\title{
RNA-Seq profiling of circular RNAs in human colorectal Cancer liver metastasis and the potential biomarkers
}

\author{
Hanchen $\mathrm{Xu}^{1}$, Chunyan Wang ${ }^{1}$, Haiyan Song ${ }^{1}$, Yangxian $\mathrm{Xu}^{2^{*}}$ and Guang $\mathrm{Ji}^{\mathrm{1}^{*}}$
}

\begin{abstract}
In this study, the secondary sequencing was used to profile circRNA expression in the tissue samples from three CRC patients with liver metastasis and three matched CRC patients. After verified some candidates in another 40 CRC and CRC-m samples by qRT-PCR, we further demonstrated that circRNA_0001178 and circRNA_0000826 were significantly upregulated in CRC-m tissues, and both of them had the potential for diagnosing liver metastases from colorectal cancer. Finally, the networks of circRNA-miRNA-mRNA base on these two circRNAs were constructed respectively. This study showed that differentially expressed circRNAs were existed between the tissue samples from colorectal cancer patients with and without liver metastasis. And also suggested that circRNA_0001178 and circRNA_0000826 may serve as a potential diagnostic biomarker for liver metastases from colorectal cancer.
\end{abstract}

Keywords: Colorectal cancer liver metastasis, Circular RNAs (circRNAs), RNA-sequencing (RNA-seq), Biomarker

\section{Main text}

Circular RNAs (circRNAs) are an important member of the non-coding RNA family following microRNAs and IncRNAs. They are characterized by the absence of covalently closed loop structures at the $3^{\prime}$ and $5^{\prime}$ ends. Base on this closed structure, circRNAs are highly stable and not easily degraded [1]. As this mechanism of circRNA as a ceRNA is revealed, this is considered to be an important way for circRNA to play a role in disease progression. CircRNA can act as a sponge to adsorb miRNA, and then regulate the expression of miRNA and its target genes [2, 3]. In addition, circRNAs have a certain specificity in different tissues and are therefore suitable as biomarkers for cancers. Recent studies have shown that circRNAs are abnormally expressed in tumor tissues, and some circRNAs as the potential biomarkers have been discovered [4-6]. However, as far as we know, there is no study on the role of circRNAs in liver metastasis from colorectal cancer. In this study, we used the secondary sequencing technology to compare the expression profile of circRNAs in the tissue samples from

\footnotetext{
*Correspondence: xuyangxian@163.com; jiliver@vip.sina.com

${ }^{2}$ Department of General Surgery, Longhua Hospital, Shanghai University of Traditional Chinese Medicine, Shanghai 200032, China

${ }^{1}$ Institute of Digestive Diseases, Longhua Hospital, Shanghai University of

Traditional Chinese Medicine, Shanghai 200032, China
}

colorectal cancer patients and colorectal cancer patients with liver metastasis. Then, we expand the sample size to verify some candidates and assess the diagnostic value of the circRNAs as the biomarkers for liver metastases from colorectal cancer. Finally, we constructed a network map based on two circRNAs and their respective potential binding miRNA-mRNAs to better investigate their potential mechanisms of action in colorectal cancer liver metastasis.

\section{Collection the tissue specimens}

A total of 40 patients were involved in this study, including 24 colorectal cancer patients (CRC) and another 16 colorectal cancer patients with liver metastases (CRC-m). Tumor tissue samples of these patients obtained from the surgical treatment at the Department of General Surgery, Longhua Hospital affiliated to Shanghai University of Traditional Chinese Medicine, China. The samples were snap froze in liquid nitrogen after separated from the human body immediately and stored at $-80^{\circ} \mathrm{C}$ before using. This study was approved by the Ethics Committee of Longhua Hospital, and the informed consent form was signed by every participant.

(c) The Author(s). 2019 Open Access This article is distributed under the terms of the Creative Commons Attribution 4.0 International License (http://creativecommons.org/licenses/by/4.0/), which permits unrestricted use, distribution, and reproduction in any medium, provided you give appropriate credit to the original author(s) and the source, provide a link to the Creative Commons license, and indicate if changes were made. The Creative Commons Public Domain Dedication waiver (http://creativecommons.org/publicdomain/zero/1.0/) applies to the data made available in this article, unless otherwise stated. 
A

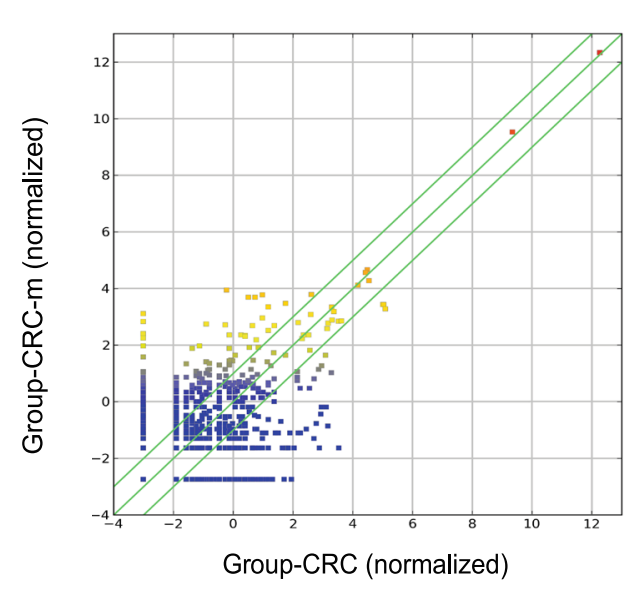

C

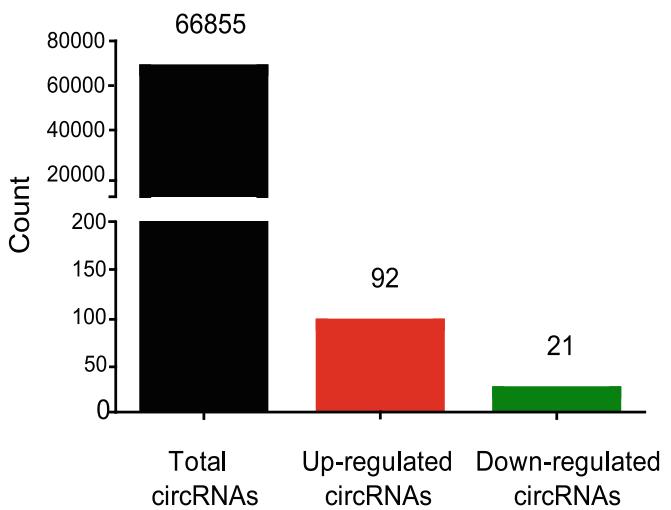

B

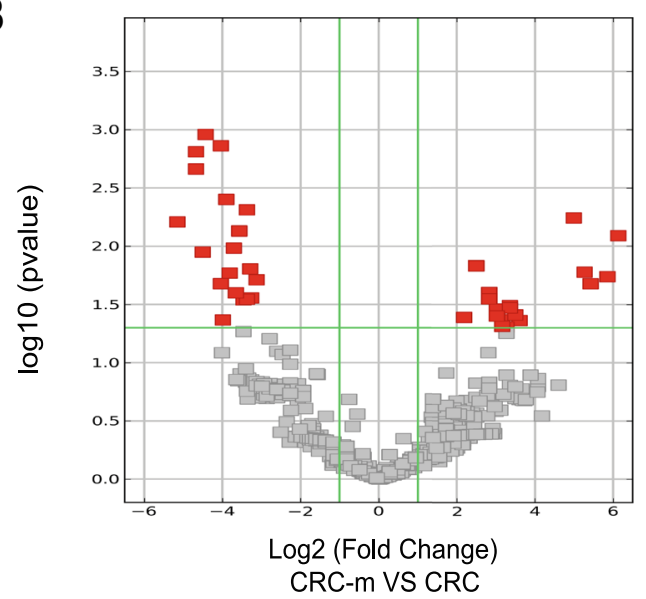

D

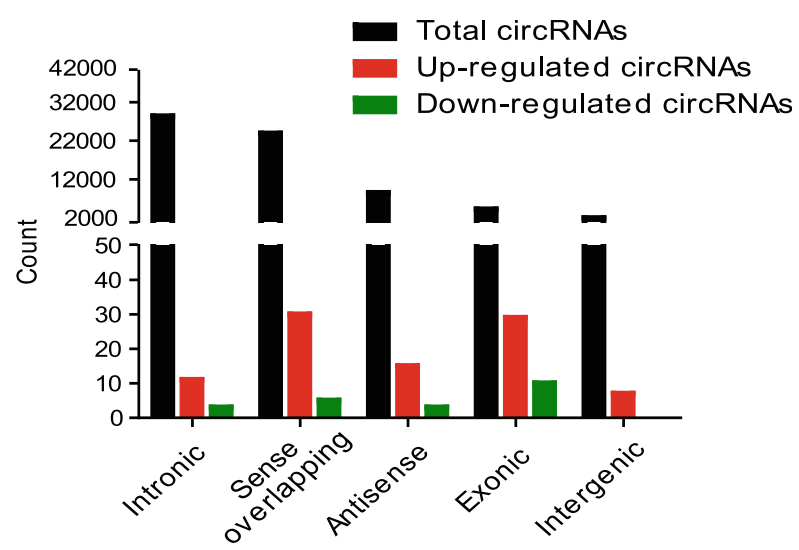

F

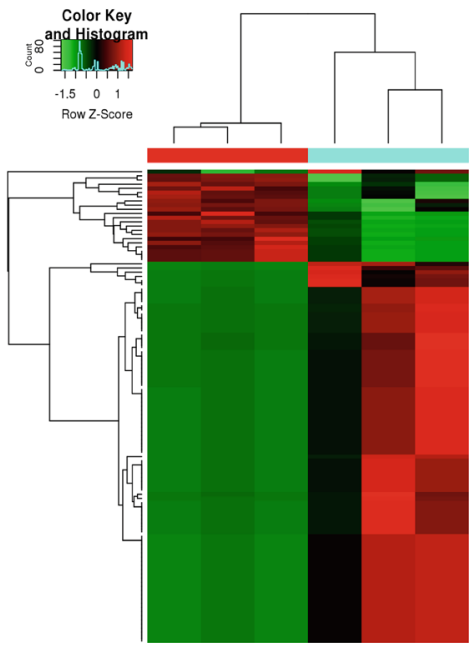

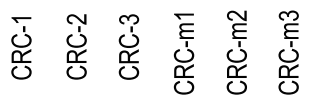

Fig. 1 (See legend on next page.) 
(See figure on previous page.)

Fig. 1 Analysis of differentially expressed circRNAs in the tissues from CRC patients with or without liver metastasis by RNA-sequencing. a The scatter plot figuratively expresses the changes in circRNA expression CircRNAs above the top green line and below the bottom green line demonstrated more than a 1.5 -fold change between the two compared groups. $\mathbf{b}$ The volcano plot was showed the expression profiling between the two groups. The vertical green lines refer to a 2.0-fold (log2 scaled) up-regulation and down-regulation, respectively. The horizontal green line corresponds to a $P$-value of 0.05 (-log 10 scaled). The red points in the plot represent differentially expressed circRNAs with statistical significance. $\mathbf{c}$ The amount of the total circRNAs and differentially expressed circRNAs. $\mathbf{d}$ CircRNAs were classified by category. e CircRNAs were distributed by located in human chromosomes. $\mathbf{f}$ Hierarchical clustering indicates differences in circRNA expression profiling between the two groups

\section{RNA-seq analysis}

The total RNA was isolated from the tissue samples using TRIzol reagent (Life Technologies, Carlsbad, CA) according to the manufacturer's instructions. RNA integrity and DNA contamination were assessed using electrophoresis on a denaturing agarose gel. Before constructing the RNA-seq libraries, the Ribo-Zero rRNA Removal Kit (Illumina,San Diego, CA, USA) and the CircRNA Enrichment Kit (Cloud-seq,USA) were used to remove the rRNA and enrich the circRNAs. The RNA-seq libraries were constructed by using pretreated RNAs with TruSeq Stranded Total RNA Library Prep Kit (Illumina, San Diego, CA, USA) according to the manufacturer's instructions. The libraries were denatured as single-stranded DNA molecules, captured on Illumina flow cells, amplified in situ as clusters and finally sequenced for 150 cycles on Illumina HiSeq $^{\text {тм }} 4000$ Sequencer (Illumina, San Diego, CA, USA)according to the manufacturer's instructions.

\section{Identification and quantification of human circRNAs}

Paired-end reads were harvested from Illumina HiSeq 4000 sequencer, and were quality controlled by Q30. The reads were aligned to the reference genome/transcriptome with STAR software and circRNAs were detected and annotated with DCC software. CircBase database and circ2Trait disease database were used to annotated the identified circRNAs. Differentially expressed circRNAs were identified by $\mathrm{T}$ test between two groups.

\section{Validation of candidate circRNAs using quantitative real-time reverse-transcription polymerase chain reaction (qRT-PCR)}

Total RNA was extracted from the tissue samples using TRIzol reagent (Life Technologies, Carlsbad, CA) and then reverse-transcribed into cDNA using the SuperScript First-Strand Synthesis System (Invitrogen, Carlsbad, CA, USA). The cDNA was used for qPCR using SYBR Green PCR Master Mix (Applied Biosystems, Foster City, CA, USA) with gene-specific primers and the results were normalized with $\beta$-actin as a control. PCR primers are listed in Additional file 1: Table S1.

Statistical analyses were performed using GraphPad Prism 7, and Student's t-test and Mann-Whitney test were used to compare two groups of independent samples, as appropriate. ROC curve analysis was performed to evaluate the diagnostic value of circRNAs in CRC-m patients compared to CRC patients. Data were presented as mean $\pm \mathrm{SD}, p<0.05$ was considered statistically significant.

\section{Prediction of circRNA-miRNA interactions}

CircRNA-miRNA interaction were predicted by popular target prediction softwares, and network was constructed by Cytoscape software. Specific predictions based on miRanda, miRDB, miRWalk, RNA22 and Targetscan databases. And for each circRNA, we showed the top 5 miRNA that potentially bind to the circRNA and the five most likely target genes to every miRNAs.

\section{Findings \\ Identification of differentially expressed circRNAs}

To identify circRNAs that were differentially expressed in CRC patients with liver metastasis, the secondary sequencing was used to profile circRNAs expression in the tissue samples from three CRC patients with liver metastasis and three matched CRC patients. The basic characteristics of the patients was provided in Additional file 1: Table S2. Under the sequencing, total 66,855 circRNAs were detected in the tissue samples. The list of total circRNAs expression profiling was shown in Additional file 1: Table S3. The scatter plot showed the variation of circRNAs expression level between CRC patients with or without liver metastasis (Fig. 1a). As was showen in volcano plot, the significant differentially expressed circRNAs between the two groups were identified with fold-changes of greater than 2.0 and $p$ value less than 0.05 (Fig. 1b). From the total 66,855 circRNAs, 92 circRNAs were significant up-regulated and 21 circRNAs were significant down-regulated (Fig. 1c and Additional file 1: Table S4). According to the source of the circRNA formation, it revealed that most significant differentially expressed circRNAs were transcribed from the exons of coding protein (Fig. 1d). Further, we classified of all differentially expressed circRNAs base on the different location on the chromosomes. All chromosomes have the expression of circRNA, and we could found the differentially expressed circRNAs except Y chromosome (Fig. 1e). Hierarchical clustering demonstrates these significant differentially expressed circRNAs (Fig. 1f). Thereinto, the top 10 circRNAs with significant up-regulation or down-regulation 


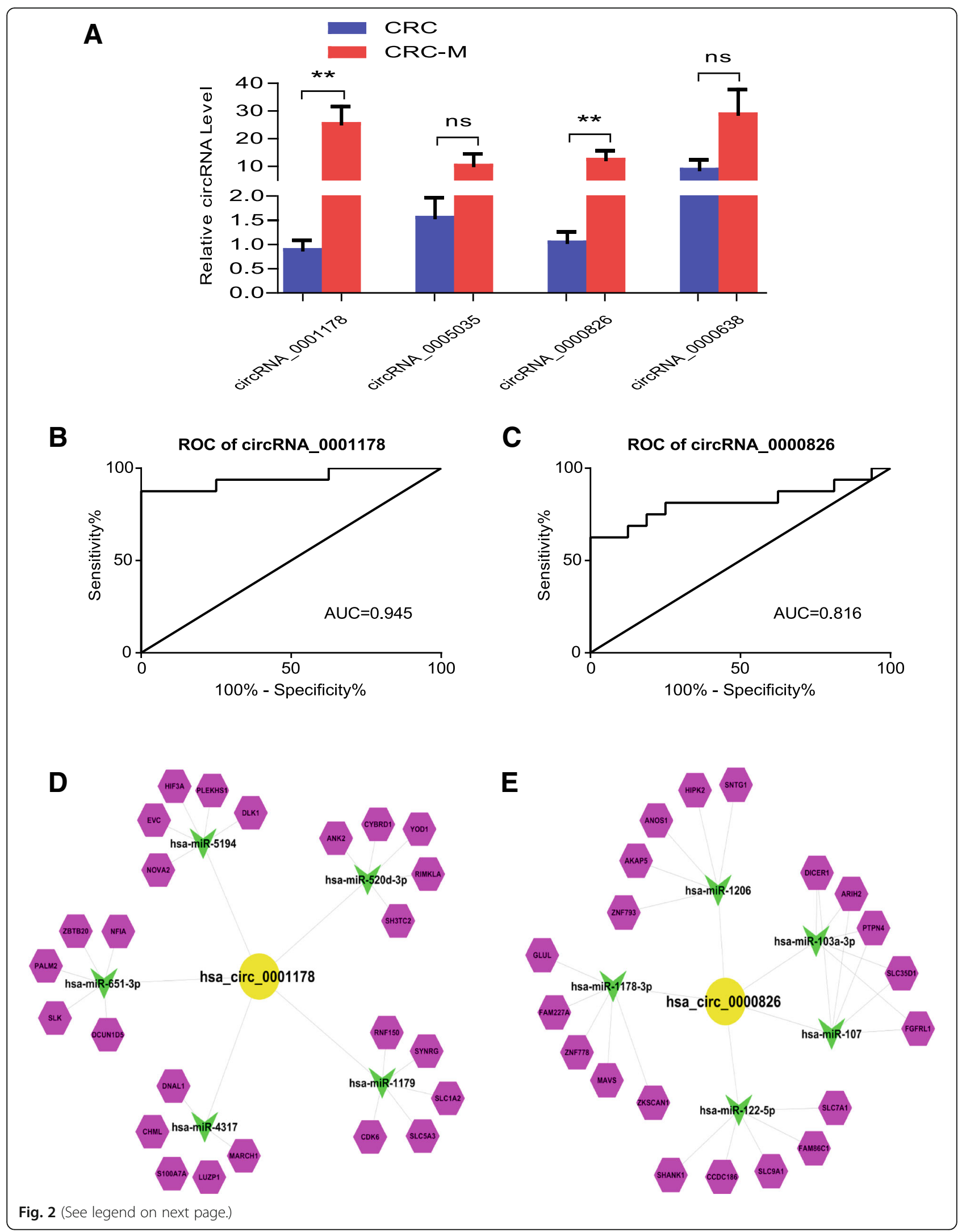


(See figure on previous page.)

Fig. 2 a Validation of the circRNAs expression by qRT-PCR. The expressions of top four up-regulated circRNAs in CRC-m samples from RNA-Seq data were evaluated using qRT-PCR in the sample from 32 CRC patients with or without liver metastasis. b, c ROC curve analysis of differentially expressed circRNAs. ROC curve analysis of circRNA_0001178 and circRNA_0000826 in CRC-m patients versus CRC patients. AUC values are given on the graphs. d, e ceRNA analysis for circRNA_0001178 and circRNA_0000826 respectively. The cytoscape software was used to show the circRNA-miRNA-mRNA interactions. Base on the miRNA prediction and bioinformatics analyses, we show the top 5 miRNAs may regulated by circRNA_0001178 (d), circRNA_0000826 (e) and top 5 target genes of each miRNA respectively

were listed in Additional file 1: Table S5, of these 20 circRNAs, the fold-changes were at least greater than 10. The circRNA-seq data and analysis suggest that some cricRNAs expressed level were different in the CRC patients with liver metastasis compared with $\mathrm{CRC}$ patients.

\section{Validation of differentially expressed circRNAs}

Considering the expression level of down-regulated circRNAs in CRC-m patients were very low and not easy to detect in disease progression, we only focus on the up-regulated circRNAs. From the list in Additional file 1: Table S5, we first selected the 4 up-regulated circRNAs which had the most significantly different expression. To verify the circRNA-seq results of these 4 circRNAs, qRT-PCR was performed in the tissue samples from 16 CRC-m patients and 24 CRC patients. As shown in Fig. 2a, the circRNA_0001178 and circRNA_0000826 were significantly up-regulated in CRC-m patients, which were consistent with previous sequencing results.

\section{ROC curve analysis of circRNA_0001178 and circRNA_0000826 in the CRC-m patients with liver metastasis}

From the validation, we confirmed the expression levels of circRNA_0001178 and circRNA_0000826 were increased in CRC-m patients. Then the ROC curve analysis was performed to assess the diagnostic value of these two circRNAs in CRC-m patients. The AUC was 0.945 (95\% CI: $0.863-1.000 P<0.001$ ) for circRNA_0001178 (Fig. 2b) and 0.816 (95\% CI: $0.656-0.977, P<0.01$ ) for circRNA_0000826 (Fig. 2c). The results suggested the potential diagnostic value for these two circRNAs in CRC-m patients.

\section{circRNA-miRNA-mRNA network prediction and analyses}

We built a circRNA-miRNA-target gene network for circRNA_0001178 and circRNA_0000826 using Cytoscape respectively. In the network map, we showed the top 5 miRNA that potentially bind to the circRNA and the five most likely target genes to each miRNA (Fig. 2d and e). From this figure, we can clearly see the potential target of circRNA_0001178 and circRNA_0000826. It gave us a clear direction to study the specific mechanism of these two circRNA in the liver metastasis of colorectal cancer.

\section{Conclusions}

In this study, through high-throughput sequencing of patient tissues and bioinformatics analysis, we obtained the differential expression of circRNAs between the tissue samples from colorectal cancer patients with and without liver metastasis. And also indicated circRNA_0001178 and circRNA_0000826 were the promising biomarkers for liver metastases from colorectal cancer.

\section{Additional file}

Additional file 1: Table S1. Sequences of the primers in this study. Table S2. Personal characteristic of patients participated in the sequencing. Table S3. CircRNA Expression Profiling in this study (please see the attached excel spreadsheet).Table S4. Differentially Expressed circRNAs between the two groups (please see the attached excel spreadsheet).Table S5. The Top 10 circRNAs with the significant upregulation or downregulation. (DOCX $19 \mathrm{~kb}$ )

\section{Abbreviations}

CDK6: Cell division protein kinase 6; circRNAs: Circular RNAs; circRNASeq: Circular RNA sequencing; CRC: Colorectal cancer; CRC-m: Colorectal cancer liver metastases

\section{Acknowledgements}

We thank Cloud-Seq Biotech Ltd. Co. (Shanghai, China) for the CircRNA-Seq service, special thanks to Ms. Yanfen Ge and Dr. Wei Li for the help during the study.

\section{Funding}

This project was supported by the National Nature Science Foundation of China (No.81874206, 81620108030).

\section{Availability of data and materials}

The datasets used and/or analyzed during the current study were available from the corresponding authors on reasonable request.

\section{Authors' contributions}

HX was responsible for collecting tissue specimen, data analysis and drafting the manuscript. CW and HS have also contributed to collecting tissue specimen and performing the experiments. There are two corresponding authors in this manuscript. YX has contributed to leading the surgery and collecting tissue specimen. GJ has contributed to study design, data interpretation, and editing the manuscript. All authors read and approved the final manuscript.

\section{Ethics approval and consent to participate}

The human cancer tissues used in this study were approved by the institute. ethical committee of Longhua Hospital affiliated to Shanghai University of Tradional Chinese Medicine.

\section{Consent for publication}

The consent forms were signed by every participant, and will be provided upon request. 


\section{Competing interests}

The authors declare that they have no competing interests.

\section{Publisher's Note}

Springer Nature remains neutral with regard to jurisdictional claims in published maps and institutional affiliations.

Received: 2 November 2018 Accepted: 27 December 2018

Published online: 10 January 2019

\section{References}

1. Memczak S, Jens M, Elefsinioti A, Torti F, Krueger J, Rybak A, et al. Circular RNAs are a large class of animal RNAs with regulatory potency. Nature. 2013;495(7441):333-8.

2. Hansen $T B$, Jensen $\mathrm{Tl}$, Clausen BH, Bramsen JB, Finsen B, Damgaard CK, et al. Natural RNA circles function as efficient microRNA sponges. Nature. 2013; 495(7441):384-8

3. Zheng Q, Bao C, Guo W, Li S, Chen J, Chen B, et al. Circular RNA profiling reveals an abundant circHIPK3 that regulates cell growth by sponging multiple miRNAs. Nat Commun. 2016;7:11215

4. Tang W, Fu K, Sun H, Rong D, Wang H, Cao H. CircRNA microarray profiling identifies a novel circulating biomarker for detection of gastric cancer. Mol Cancer. 2018;17(1):137.

5. Lu C, Shi X, Wang AY, Tao Y, Wang Z, Huang C, et al. RNA-Seq profiling of circular RNAs in human laryngeal squamous cell carcinomas. Mol Cancer. 2018;17:86.

6. Zhang S, Zeng X, Ding T, Guo L, Li Y, Ou S, et al. Microarray profile of circular RNAs identifies hsa_circ_0014130 as a new circular RNA biomarker in non-small cell lung cancer. Sci Rep. 2018;8(1):2878.

- fast, convenient online submission

- thorough peer review by experienced researchers in your field

- rapid publication on acceptance

- support for research data, including large and complex data types

- gold Open Access which fosters wider collaboration and increased citations

- maximum visibility for your research: over $100 \mathrm{M}$ website views per year

At $\mathrm{BMC}$, research is always in progress. 